\title{
EL PROBLEMA DE LA RACIONALIDAD EN LA ECONOMÍA NEOCLÁSICA
}

\author{
JOSÉ ATILANO PENA LÓPEZ*
}

Resumen: El presente trabajo analiza los rasgos fundamentales del denominado homo oeconomicus, esto es, las bases antropológicas del razonamiento económico generalizado en la mainstream, para poner de relieve las lagunas presentes en esta construcción teórica, en particular su incapacidad para recoger la dimensión social y ética del hombre. Partiendo de esta crítica, sistematizaremos las diversas propuestas alternativas, haciendo hincapié en la necesidad de integrar las dimensiones "extraeconómicas» dentro del razonamiento económico, concretamente, las referentes al comportamiento ético. En este sentido nos detendremos especialmente en las denominadas propuestas humanista y austriaca. Finalmente, y en razón de lo anterior, se proponen una serie de cambios necesarios en el análisis de la toma racional de decisiones por los agentes en Economía.

Palabras clave: homo oeconomicus, antropología económica, ética, escuelas heterodoxas.

Abstract: The present work analyses the fundamental characteristics of homo oeconomicus, that is to say, the anthropological bases of the generalised economic mainstream reasoning, to demonstrate the deficiencies of this theoretical construction, particularly its incapacity to gather the social and ethical dimension of man. Considering these weaknesses, we will systematise the diverse alternative proposals, insisting on the necessity to integrate the "extraeconomic» dimensions within the economic reasoning, concretely, the ethical behaviour. In this sense we study specially the Humanist and Austrian schools. Finally, a set of necessary changes in the process of analysis of economic reasoning are proposed, trying to introduce anthropologic realism in economic models.

Key words: homo oeconomicus, economic anthropology, ethics, heterodox perspectives.

Clasificación JEL: B41, B50, B52, B53.

* Universidade de A Coruña. Mail: atilano@udc.es. 
«El hombre económico se comportaría de este modo en una situación habitual:

— ¿Dónde está la estación de ferrocarril? —le pregunta alguien. - Allí — contesta, señalando la estación de correos-. Y de paso, ¿podría echarme esta carta en el buzón?

- Sí - contesta el otro, decidido a ver si tiene algo valioso para abrirla...»

A.K. SEN (1977, p. 324)

\section{INTRODUCCIÓN: ECONOMÍA Y SIMPLIFICACIONES ANTROPOLÓGICAS}

Las orientaciones críticas en Economía constantemente acusan de irrelevancia a la teoría económica convencional en lo que respecta a los «problemas reales» (Wiles y Routh, 1984), ya que, cuando éstos entrelazan hechos antropológicos, sociales y políticos, son obviados bajo la consideración de no-económicos. Esto da lugar, por una parte, a un notable aislacionismo de la Ciencia Económica y, por otra, a cierto imperialismo metodológico en el estudio de aspectos que deberían ser interdisciplinares.

Una de las razones de este aislacionismo radica en que la teoría económica no hace justicia a las dimensiones culturales e históricas de su materia. Concretamente, la mainstream ha sustituido un estudio de la complejidad de la naturaleza humana por el análisis de un simple y poderoso imaginario al que denominamos homo oeconomicus (Hollis y Nell, 1975, pp. 10-35).

Si nos retrotraemos a las raíces de este problema, las encontramos en la propia concepción de la racionalidad. Tal y como recogía H. Simon (González, 2004), el status del concepto de racionalidad en el ámbito de las ciencias sociales se puede sintetizar en tres puntos. Por una parte, hay una fascinación por la teoría de la utilidad esperada pese a que se descubre un creciente número de pruebas empíricas divergentes acerca de la conducta humana. Este hecho ha conducido, por otra parte, a la 
introducción de hipótesis ad hoc en forma de supuestos específicos sobre los límites de la racionalidad y a una creciente preocupación por los estudios empíricos que ponen en cuestión las derivaciones de la teoría neoclásica ${ }^{1}$. Sin embargo, pese a que se han dado pasos en el ámbito de la psicología cognitiva para formular una teoría sobre la toma de decisiones fundada empíricamente, la incursión del razonamiento formal propio de la Economía sobre las restantes ciencias sociales no ha comenzado a decaer.

Este problema, además, es especialmente relevante para la práctica de la Política Económica. Uno de los objetos esenciales de la materia es la acción colectiva o cómo se organizan las agrupaciones humanas en el desarrollo de tareas comunes. En general, los seres humanos presentamos una clara tendencia hacia el «exceso de cooperación» frente a lo que podría derivarse de los supuestos sobre la racionalidad individual de la Ciencia Económica. Precisamente, la ética entendida como esta voluntad de cooperación es una de las matrices del funcionamiento del mercado y del desarrollo económico (Zamagni, 1989, pp. 65-77; 1994, pp. 281-296).

\section{UNA DESCRIPCIÓN ELEMENTAL}

El homo oeconomicus es optimizador, inmutable, irreflexivo en lo que respecta a los fines ${ }^{2}$, egoísta ${ }^{3}$ (no-tuista) desprovisto de toda identificación grupal y primordialmente materialista. En otras pala-

1 Éste es el caso de los trabajos de dos recientes premios Nobel (Selten, 1994; V. Smith, 1998).

2 Es decir, que carece de racionalidad substantiva. Sin embargo, esta aparente ausencia no deja de encubrir una opción por una racionalidad substantiva encubierta en la propia definición. El hombre es finalista pero los fines ya están predeterminados en la teoría.

${ }^{3}$ En el sentido de que se despreocupa por la situación del otro. El término egoísmo resulta especialmente ambiguo. La independencia de las funciones de uti- 
bras, se trata de un ser racional en el sentido instrumental que posee un conjunto de preferencias totalmente ordenado e inmutable, información perfecta, una inmaculada habilidad de cálculo, y que busca maximizar su utilidad definida en un sentido individualista y material. Por tanto, el hombre es definido como una especie de "cazador de gangas», una definición tan simple que resulta inaceptable ante la menor introspección y especialmente en términos morales (Sen, 1985, pp. 1-19) ${ }^{4}$.

Metodológicamente eludimos la debilidad de estos supuestos considerando irrelevante el realismo de los supuestos, de forma que el conflicto no es entre la realidad y la teoría, sino entre la capacidad explicativa de una teoría frente a otra (Friedman, 1956, pp. 3-43). Así, una aparente mayor capacidad explicativa del funcionamiento del sistema económico valida incluso la falta de realismo antropológico, de tal modo que el hombre económico sólo es una representación parsimoniosamente útil que genera un conjunto consistente de hipótesis hasta cierto punto plausibles en contrastaciones empíricas.

La descripción anterior es una consecuencia lógica del «individualismo metodológico» típico de la economía. Fruto del cual los economistas explican los procesos sociales como el resultado de la interacción de decisiones individuales, por lo que no es posible comenzar la construcción de la teoría sin haber establecido antes los fundamentos de la motivación humana (Boland, 1982). Esto nos remite al estudio de un doble problema: las preferencias y la racionalidad en Economía.

lidad individuales es recogida mejor por la expresión no-tuismo. En adelante utilizaremos indiferentemente ambos términos; sin embargo, éste último es más apropiado.

4 A. Sen, «The moral standing of the market», en E.F. Paul y otros (eds.), Ethics and Economics, Oxford, Blackwell, 1985, 1-19. Históricamente, el origen de concepto de homo oeconomicus se remonta hasta J.S. Mill (no en Smith); éste es el primer autor que opta por la extracción de una serie de características de los comportamientos humanos para realizar un constructo abstracto. Sobre el tema puede verse Blaug (1980, 83-120). 
Comenzaremos por esta última para, posteriormente, reconsiderar la ética implícita en la Economía o el problema de las preferencias.

\subsection{La racionalidad en Economía}

La condición del hombre como decision-maker se fundamenta en tres presupuestos básicos. Los comportamientos deben ser siempre sistemáticos (en la misma situación el comportamiento será el mismo), adaptativos a las circunstancias (no son respuestas ajenas al entorno) y discernibles (siguen un esquema racional de respuesta asociado a la existencia de unos fines concretos). Expresado de un modo muy sintético, cada agente construye un modelo racional del medio en el que se encuentra, consistente con la información de que dispone, y selecciona los medios precisos para la consecución de los objetivos que se plantea.

Así, la concepción generalizada del homo oeconomicus supone un proceso de decisión que se limita al cálculo y a la optimización. Bajo esta perspectiva, cualquier alteración de comportamiento se explica como una nueva respuesta óptima a la modificación del entorno, pero no en función de un cambio en las preferencias.

Ahora bien, si entendemos que la racionalidad se caracteriza primordialmente por la búsqueda inteligente de fines apropiados, la noción de racionalidad en Economía se limita a los aspectos instrumentales o prácticos abandonando los cognitivos y valorativos. La tradición filosófica desde Kant señala la existencia de tres contextos de elección: los relacionados con la creencia (razón epistémica), los correspondientes a la acción (razón práctica) y los concernientes a la evaluación (razón evaluativa) (Rescher, 1988, pp. 2-4). Por consiguiente, podemos hablar de una racionalidad referente a las creencias, que lleva a acep- 
tar determinadas tesis o planteamientos; una racionalidad instrumental o práctica que determina qué actos se deben realizar, y, finalmente, una racionalidad evaluativa que fija qué debe ser valorado. Según el análisis característico de la Elección Racional, se asume la existencia de una racionalidad práctica y una cognitiva, aunque ésta sea deficiente, de modo que, dados sus deseos y creencias, definidos como inmutables, el individuo se limita a optimizar. Pero no se hace ningún tipo de mención al tercer modo de racionalidad, ya que los fines están dados de antemano y no son cuestionados. Concretamente, en el consenso neoclásico se concibe una racionalidad instrumental que busca maximizar las expectativas subjetivas esperadas, siendo juzgadas exclusivamente en función de los resultados obtenidos (la utilidad), de modo que es ajena a la racionalidad de los fines o evaluativa. Consecuentemente, las discusiones abiertas sólo son desde el punto de vista de medios, abandonando los aspectos cognitivos y evaluativos (Simon, 1983, pp. 7-10; González, 2002, pp. 150 y ss.).

Vamos a revisar estas dos dimensiones:

En lo que respecta a la racionalidad cognitiva, nos puede resultar particularmente esclarecedor el planteamiento de $\mathrm{H}$. Simon. Este autor propugna la existencia de una racionalidad limitada (bounded rationality) en la elección de los medios para alcanzar los fines, reconociendo las restricciones subjetivas que existen en este proceso fuera del control de los agentes, totalmente ausentes en los estudios de Economía. Así, debemos distinguir entre racionalidad sustantiva y racionalidad procesual. La primera de ellas forma parte del estereotipo del homo oeconomicus y es no limitada y se encontraría determinada por las características del entorno o restricciones objetivas, de forma que el actor económico tiene como meta la maximización del beneficio o utilidad y poderes de cálculo ilimitados. La simpleza de estas propuestas nos llevaría a suponer un absurdo como que los individuos perciben el mundo tal cual es. La racionalidad 
de procedimiento o limitada, en cambio, va a depender del proceso que se genera, en lugar de descansar en la obtención de metas dadas, procedimiento limitado en lo que respecta al conocimiento y a las capacidades individuales; por tanto, hay una distinción entre el mundo real y la percepción que el agente tiene de ese mundo. Consecuentemente, frente a la mainstream en Economía, en la que una racionalidad perfecta y omnisciente se ajusta a un entorno externo, la racionalidad procesual es adaptativa y ha de descubrir la estrategia adecuada, un proceso complejo donde la capacidad de predicción se ve fuertemente cuestionada (Simon, 1976).

Cuando hablamos de racionalidad limitada también nos referimos al hecho de que las elecciones de los agentes no están exclusivamente determinadas por un objetivo general como pudiese ser la maximización del nivel de utilidad y por el entorno que rodea al sujeto, sino también por el conocimiento del mundo de aquellos que toman las decisiones, de su habilidad para recordar y extraer conclusiones, de la capacidad para afrontar la incertidumbre, incluso de sus capacidad para integrar sus deseos en competencia (Simon, 2000, pp. 25-39). La racionalidad es limitada por la sencilla razón de que estas habilidades se encuentran severamente condicionadas. Por consiguiente, la conducta racional está tan severamente limitada por el entorno interno o los procesos personales como por el externo.

Frente a las tradicionales teorías sustantivas, centradas en el estudio de la calidad del resultado de un proceso de toma de decisiones, la racionalidad limitada se fijará en la calidad de los procesos de toma de decisión, con lo que se hace necesario integrar la psicología de la toma de decisones en el estudio para reemplazar a los razonamientos de caja negra tradicionales en Economía, donde sólo es considerada la existencia de una meta y de un entorno concreto.

No obstante, aunque considerar la perspectiva del carácter limitado y adaptativo de la razón nos permite aproximar pro- 
cesos reales, todavía no hemos abandonado el principal problema de la ciencia económica. Dado que la razón sigue siendo totalmente instrumental, no podemos decidir a dónde vamos, sino que ahora sabemos que tanteamos el modo de llegar a un lugar definido con anterioridad. Simplemente, habríamos introducido realismo en el modelo de maximización de la utilidad esperada al considerar una perspectiva conductual (behavioral model) en un mundo más complejo ${ }^{5}$. Por tanto, en esta propuesta los fines siguen estando dados, pese a que la razón también es capaz de evaluar estos fines y optar entre ellos.

Retornando a la dimensión evaluativa, la racionalidad no sólo depende de lo que queremos, sino de lo que debemos querer $\mathrm{o}$, dicho de otro modo, de los fines que es aconsejable escoger en las circunstancias imperantes. De este modo, contrariamente a la perspectiva generalizada, que limita la racionalidad a la elección ante fines dados, la racionalidad es la búsqueda inteligente de fines apropiados (Rescher, 1988, p. 1), de forma que es precisa una evaluación racional de los fines (racionalidad evaluativa), con lo que los aspectos positivos y normativos están íntimamente imbricados.

Los trabajos de R. Selten y de las orientaciones conductuales tratan de introducir una dimensión evaluativa y una perspectiva procesual en el análisis de la racionalidad. Los individuos en estos trabajos parten de la información a la que tienen acceso sobre la que aplican un análisis cualitativo (racionalidad epistémica), estos agentes también disponen de unas metas formadas por un proceso de discernimiento individual (racio-

5 Los estudios conductuales son ya compleja rama de investigación con diversas derivaciones (psicología, teoría de juegos, microeconomía...); todas ellas tratan de explicar las decisiones económicas que los individuos adoptan en la práctica, especialmente cuando éstas no coinciden con los presupuestos de la teoría económica convencional. Es más, tratan de reemplazar al homo oeconomicus por modelos más complejos tomados de la psicología cognitiva, donde los individuos se ven influidos por la aversión al riesgo, disonancias cognitivas y sesgos derivados de sus grupos de pertenencia (Camerer, 1997, pp. 167-188). 
nalidad evaluativa) que no tiene por qué identificarse con la maximización de la utilidad individual, para finalmente, conformar una actuación pública o los medios para el logro de ese fin (racionalidad práctica). Concretamente, este autor ha encontrado un buen ejemplo de racionalidad evaluativa en el denominado juego de solidaridad. En este caso, los jugadores presentan un modo de racionalidad distinto del instrumental, que está basado en el valor de la solidaridad (Selten y Ockenfelds, 1998, pp. 517-539). En otros juegos de laboratorio también se aprecia la presencia de valores como solidaridad, equidad, etc., que sirven para modular los fines de los agentes, de forma que la maximización de la utilidad no tiene por qué ser la estrategia dominante y la elección humana está dirigida por un complejo de motivaciones que se basan en la anticipación de consecuencias (placer o dolor) para quien elige y se convierte en altruista en la medida en que anticipa el placer o dolor de otras personas (Simon, 1993, pp. 156-161) .

Esta propuesta se basa, por tanto, en una imagen del ser humano más amplia y recurre a la noción de satisfacción para eliminar el restrictivo concepto de maximización de la utilidad, un punto de vista más acorde con los resultados de la folk psicology, ya que no requiere ni la maximización ni la coherencia de las preferencias

\subsection{La ética de la racionalidad económica}

Como vimos, la Ciencia Económica se ocupa de los agentes eligiendo racionalmente; no obstante, las generalizaciones sobre la toma de decisiones son igualmente proposiciones sobre el modo en que la gente debe realizar esas elecciones. La racio-

6 Entre las experiencias realizadas destacan las extensas series de contrastaciones empíricas realizadas sobre el popular «Dilema del Prisionero». 
nalidad es en sí misma una noción normativa pero es más dudoso que pueda ser considerada una noción ética. Sin embargo, este confusionismo está presente en el uso de los términos racionalidad y moralidad. Cuando calificamos algo como irracional no lo estamos simplemente describiendo sino que lo estamos condenando, con lo que esta afirmación tiene un carácter normativo. Consecuentemente, la Elección Racional introduce elementos de teoría moral en la economía positiva que acaban derivando en la propia economía normativa (Davidson, 1980, pp. 3-20).

Para un economista, una elección es considerada racional cuando es el producto de un conjunto racional de creencias y preferencias. Un agente es racional en la medida en la que elige aquello que prefiere entre las cosas que puede obtener, siempre y cuando sus preferencias sean completas y transitivas, o, lo que es lo mismo, que puede ordenar cualquier conjunto de bienes de forma coherente (ordinal representation theorem). Ahora bien, la Teoría de la Elección Racional no dice nada sobre lo que la gente quiere, por lo que le es indiferente si un individuo toma en consideración la utilidad de los otros. Por tanto, en cuanto teoría no considera lo que los individuos deben elegir sino que trata de establecer un modelo sobre cómo los individuos eligen.

Como consecuencia, las explicaciones en Economía sólo inciden en los aspectos vinculados con las preferencias, por el simple hecho de que en los relacionados con la racionalidad de la toma de decisiones se supone que el agente tiene conocimiento perfecto. Si a esto le añadimos que un individuo racional y egoísta (self-interested) siempre prefiere lo que es mejor para sí mismo a lo que supone que es peor, el bienestar de un individuo se ve únicamente determinado por el modo en que sus preferencias son satisfechas. Así, la teoría económica ortodoxa identifica la satisfacción de preferencias con el bienestar, lo cual, desde el punto de vista filosófico, es, cuando menos, 
controvertido y con claras consecuencias en la economía normativa.

En definitiva, la Economía consagra un principio de benevolencia mínima: permaneciendo todo lo demás igual; algo es moralmente bueno si los individuos mejoran en su bienestar. Esta cláusula ceteris paribus la convierte en una afirmación incontrovertible, dado que se trata de una afirmación tautológica traducible por «permaneciendo todo lo demás igual es moralmente bueno estar mejor», pero no dice nada en absoluto sobre problemas como incrementar o no la desigualdad. Simplemente podemos afirmar que cualquier mejora paretiana es moralmente aceptable (Haussman y Mcpherson, 1993, pp. 671-731).

Sin embargo, deberíamos responder a aquello que parece otorgar a los economistas una cierta autoridad moral sin el más mínimo recurso a la reflexión ética. La razón la hemos mencionado anteriormente, los economistas no consideran controvertida la identificación entre bienestar y satisfacción de preferencias, sino una parte de su visión de la racionalidad. Sólo sería factible identificar bienestar y satisfacción de preferencias si se verificase que los individuos son racionales, autointeresados y perfectamente informados y que las preferencias individuales no pueden ser manipuladas ni deformadas hacia expresiones erróneas, lo cual sabemos que no es cierto. Nuevamente estamos ante el problema de la conciencia rectamente formada. Es la concepción del bienestar como preferencia-satisfacción la que diluye la moral en el ámbito de la Economía. A título de ejemplo, esta simplificación nos llevaría a aceptar como válido el conocido argumento para justificar el robo de un banco de W. Sutton. Simplemente, allí estaba el dinero, o incluso el aceptar que la maximización de beneficios sea un buen criterio para contaminar un río o el recurso al homicidio siempre y cuando los beneficios superen a los costes derivados. 
No obstante, nos resta por analizar un problema, si cabe más grave, dentro de este ámbito, la autonomía de las preferencias individuales (Becker y Stigler, 1977, pp. 76-90). Nuevamente, la razón de este restrictivo supuesto es la simplificación. Los cambios en las alternativas o en el medio son fácilmente cuantificables en tanto que los cambios en las preferencias o características psicológicas no pueden ser objetivamente medibles. Introducirlos en el modelo impediría identificar el impacto de los cambios en el entorno sobre el comportamiento y lo haría difícilmente comprensible. Expresado con una analogía matemática, una ecuación con dos incógnitas es indeterminada, luego conviene fijar uno de los términos para alcanzar una solución en el otro. De este modo, se abandonan sistemáticamente los aportes de las restantes disciplinas conductuales, en particular de la psicología y de la antropología, con el fin de dotar de mayor consistencia a los modelos explicativos. Éste es probablemente el gran agujero metodológico de la teoría, ya que la noción de preferencia se transforma en el auténtico deus ex machina en el que integra desde las opiniones morales hasta sus gustos culinarios.

Este problema no es exclusivo de la Economía Neoclásica, sino que se encuentra igualmente presente en algunas orientaciones críticas que optan por simplificaciones antropológicas de signo distinto a la economía ortodoxa (Cason, 1990, p. 10). Podemos establecer una doble distinción dentro de éstas, dependiendo de su perspectiva sobre el sujeto:

(i) Por una parte, las orientaciones de tradición marxista adoptan como punto de partida una reducción del conjunto de alternativas posibles, de modo que los individuos se ven abocados de forma determinista a actuar en virtud de una posición social o de la pertenencia a un determinado grupo, lo que constituye el entramado de la lucha de clases. Bajo esta perspectiva los sujetos no se encuentran 
con un continuum, sino con un conjunto discreto de posibilidades, y el determinismo de las preferencias es sustituido por el de los condicionantes sociales.

(ii) A su vez, las aproximaciones institucionalistas niegan la existencia de alternativas reales, en tanto que los sujetos no pueden plantearse hipótesis sobre lo que desconocen, con lo que su comportamiento es esencialmente inercial y tienden a repetir las elecciones realizadas con anterioridad. La racionalidad humana partiría entonces de una tabula rasa sobre la que las preferencias son gravadas por un sistema de programación cultural. Bajo esta perspectiva no tendrían lugar las respuestas adaptativas a los cambios en el medio o éstas sólo serían discretas y ante fuertes alteraciones del entorno. En este sentido, tiene un especial relieve el estudio de las relaciones de poder, ya que la capacidad de castigar o recompensar desplaza la desobediencia o los comportamientos arriesgados de los abanicos de elección y los orienta en una determinada dirección mediante la fijación de las preferencias.

\subsection{El sistema de Equilibrio General}

En último término, las simplificaciones antropológicas sobre la capacidad de elección del hombre, que vengo señalando, son el soporte adecuado para el sistema de Equilibrio General. Éste se presenta como un modo de resolución de conflictos sobre bases exclusivamente individuales y sin necesidad de recurrir a sistemas de organización jerárquica para el mantenimiento del orden. De hecho, como ciencia enraizada en el periodo ilustrado, la Economía participa de todo el movimiento en pro del individualismo y en la defensa del establecimiento de un cuerpo de libertades básicas e inalienables (Goldsmith, 1985). A su 
vez, la revolución newtoniana, al generar una comprensión del universo como un todo interrelacionado y en equilibrio de fuerzas contrapuestas, puso las bases del paradigma mecanicista sobre el que se asienta la metodología clásica en Economía, de tal modo que la tópica «Mano Invisible» smithiana es perfectamente interpretable como la traducción de este paradigma en el sistema socio-económico. Posteriormente, el utilitarismo benthamiano supuso la base filosófica sobre la que construir la traducción matemática de este sistema; es más, sin éste no habría podido desarrollarse la revolución marginalista que acabó por estrechar el foco de estudio de la Economía sobre la toma de decisiones descentralizada y con un fuerte componente matemático (Arrow y Debreu, 1954, pp. 265-290), abandonando aspectos cruciales introducidos en su momento por los clásicos.

Sin embargo, la elegancia teórica del concepto no es ajena a importantes fallos. Por ejemplo, para que el Equilibrio General sea eficiente es preciso que toda la coordinación sea realizada a través de los mercados, lo que introduce fuertes restricciones. Cuando estas condiciones no se satisfacen - es decir, ante un problema de externalidades- el sistema no sería válido, lo que implica que el Equilibrio General sólo sea aplicable a bienes privados y no a los bienes públicos. De igual manera, el Equilibrio General requiere que cada individuo sólo vea afectada su utilidad por su propio consumo, que sea puramente notuista. En la medida en que los sujetos viesen afectada su función de utilidad por la de otros individuos, se destruirían los supuestos básicos del sistema7.

7 Este razonamiento, comúnmente asumido, se ha revelado como falseable. La existencia de interdependencia entre las funciones de utilidad puede ser compatible con el sistema de Equilibrio General, bajo dos condiciones: el conocimiento de las funciones de utilidad ajenas y la atenuación progresiva de la interdependencia en razón de la distancia. Al respecto, puede verse Dimand y Dimand (1991, pp. 61-75). 
Si a los problemas anteriores les añadimos los asociados a la existencia de una forma deficiente de conocimiento e información y la perfectibilidad de éste (aprendizaje), se pone especialmente de relieve el carácter estático del modelo, que asume que toda la información está disponible inicialmente ${ }^{8}$.

El hecho es que resulta imposible anticipar toda la información y en estas circunstancias, cuando las decisiones presentes tengan consecuencias futuras, un individuo completamente racional debería considerar todas sus posibles repercusiones; por consiguiente, en un sistema económico fuertemente interdependiente se requeriría un complejo análisis a todas luces inabarcable. En la práctica, los individuos obvian un amplio conjunto de posibilidades, adoptando comportamientos inerciales, fruto de un proceso de aprendizaje centrado sobre comportamientos pasados, y esperados y los fallos se concentran en su incapacidad de anticipación de los comportamientos de otros sujetos.

Ahora bien, el fallo más evidente va en otro sentido: el agujero antropológico del hombre económico convencional al que venimos aludiendo hace inexplicable el hecho humano más relevante, la persistencia de colusión entre individuos y las relaciones de solidaridad (Schelling, 1960) ${ }^{9}$.

\footnotetext{
${ }^{8}$ Introducir el riesgo en la teoría facilita la incorporación de la inexistencia de información perfecta. El agente incorpora la utilidad esperada descontando las consecuencias de sus acciones en función de su probabilidad. Esto le exige disponer de una distribución de probabilidades completa, aunque sólo sea subjetiva, e igualmente debería considerar los costes de información y procesado de la misma. Pese a ello, los aspectos básicos del modelo permanecen.

${ }^{9}$ Las derivaciones de este problema se prolongan en infinidad de agujeros de la Teoría Económica, como la fundamentación microeconómica de la rigidez en precios y salarios, la ilusión monetaria. A título de curiosidad, merece la pena recordar el concepto de invisible handshakes citado en la obra de A. Okun (1981). Éste es también el caso del conocido análisis de Akerloff sobre la tendencia a formar agrupaciones de fidelidad entre congregaciones con raíces personales comunes en (Akerlof, 1983, pp. 54-63). En todos ellos se intenta responder a comportamientos inexplicables desde el estricto imaginario de la economía.
} 
Tomando algunos ejemplos cercanos, la creciente preocupación por la productividad ha puesto de relieve el carácter discrecional del esfuerzo y la dedicación dentro de las organizaciones ${ }^{10}$. El homo oeconomicus no es un individuo en quien pueda confiarse, puesto que siempre juega con la posibilidad de sacar ventaja de cualquier situación, los únicos incentivos posibles son de naturaleza monetaria y siempre que exista un control directo. Sin embargo, múltiples instituciones optan por la persuasión moral, reforzando los comportamientos altruistas por medio de un sentido de compromiso con respecto a la organización.

Si tomamos en consideración ahora la operativa de los mercados, éstos funcionan de modo radicalmente diferente al modelo walrasiano. La principal razón de esta disonancia reside en la dimensión espacial de las transacciones. Un acuerdo se asocia a un encuentro entre individuos y presenta una causalidad múltiple, puesto que pueden ser derivados tanto de transacciones comerciales como subproductos de otras actividades sociales. Además, la negociación es un proceso en el cual cada parte trata de influir en la percepción del coste de oportunidad de la otra parte, de modo que el proceso termina cuando ambos consideran que los costes de prorrogar la negociación para manipular las percepciones de la otra quedan contrarrestados por los beneficios de concluir el acuerdo inmediatamente. Como consecuencia, la fijación de precios consume más tiempo y se ve influida por más factores que los supuestos por el mercado walrasiano. Es más, no puede hablarse de la existencia de un precio único, sino de una fuerte dispersión de precios en transacciones simultáneas, condicionadas por las distancias físicas y sociales.

Junto a lo anterior, la ejecución de los contratos resultantes puede conllevar una serie de problemas, al existir dificultades

10 Éste es el típico problema de la teoría de la Agencia, como, ejemplo, ver Arrow (1985, pp. 37-55). 
de sincronización entre recepción y pago, lo que eleva el riesgo de incumplimiento (default risk), a lo que hay que añadir la asimetría informativa. Para los estadios mencionados de búsqueda y negociación no existen vías de reducción de costes por incentivos contractuales; por tanto, los costes de transacción asociados a cualquier intercambio son típicamente costes fijos independientes del volumen y valor del comercio, de forma que muchos mercados no son factibles porque las ganancias derivadas del comercio son insuficientes para cubrir dichos costes. En este sentido, la confianza generada entre los miembros de una institución estable desempeña un papel crucial como base de una cooperación espontánea, y la ética, a su vez, se convierte en indispensable al desarrollar relaciones de compromiso con respecto a su institución de referencia o grupo social.

Esta debilidad se traslada también hacia otras áreas de la teoría económica convencional, donde las principales orientaciones han buscado alternativas a los planteamientos tradicionales. El caso más destacado es el de la Economía del Desarrollo y la persistente dificultad para explicar las diferencias interregionales en los niveles de vida y las tasas de crecimiento. Muy difícilmente las disparidades son exclusivamente un producto de las dotaciones factoriales y el dominio tecnológico, ya que factores como los de la cultura, la ética o la religión, y otros de difícil cuantificación, juegan un papel igualmente clave.

Por tanto, desde el punto de vista de la mainstream en Economía el homo oeconomicus se asimila al comportamiento de un decision-maker individualista que constantemente reinventa su entorno, carece de un pasado común y de una pertenencia grupal, cuando estas dimensiones se revelan como constitutivas del ser humano y de la actividad económica. Expresado de otro modo y recogiendo las categorías utilizadas por M. Buber, la Economía sufre una notable incapacidad para tomar en consideración los a priori de las relaciones económicas (Gui, 2000, pp. 139-169; Macnail, 1985, pp. 541-546). Frente a ésta, las restan- 
tes ciencias sociales, en particular la sociología, adoptan perspectivas orgánicas en las que el individuo pasa a ocupar un papel secundario y las normas se vuelven prioritarias a las preferencias. Esta visión orgánica va a destacar las relaciones de largo plazo al margen del mercado que existen entre individuos, tanto en actividades de producción como de consumo. Lo evidente en este caso es que la adopción de un «individualismo metodológico» no debe obviar la importancia de las agrupaciones sociales, la ética de las mismas y sus instituciones, en la coordinación económica, al igual que la preocupación por los intercambios no debe ocultar el análisis de la naturaleza de los «encuentros y acuerdos» entre individuos, así como la generación y el consumo de bienes relacionales en dichos encuentros.

Este problema sugiere el planteamiento de una agenda de investigación alternativa, centrada en la explicación de la evolución histórica de las instituciones económicas y del impacto de dicha evolución en la actuación económica (Hodgson, 1988) ${ }^{11}$.

\section{LA NATURALEZA HUMANA \\ Y EL PROBLEMA DEL HOMO OECONOMICUS}

A modo de síntesis, puede ser interesante realizar un recorrido por los principales puntos de confrontación entre las ciencias conductuales, en particular la psicología, y el paradigma anteriormente expuesto.

Un importante conjunto de investigaciones sobre el comportamiento real de los agentes económicos ha observado la existencia de desviaciones consistentes respecto a los supuestos

${ }^{11}$ Los estudios de corte institucional han ido cubriendo parte de esta agenda de investigación pendiente; concretamente, este autor va a considerar la capacidad de las instituciones sociales para moldear comportamientos y preferencias, superando los límites de la racionalidad del homo oeconomicus. 
tradicionales del homo oeconomicus (Henrich, 2001, pp. 73-79). Este hombre económico constituye una caricatura del comportamiento real ${ }^{12}$. Por ejemplo, comúnmente las preferencias de los individuos no respetan el supuesto de transitividad, continuidad (Loomes y Taylord, 1992, pp. 357-365), irreversibilidad (Knetch, 1992, pp. 129-139) e interés particular (Fehr y Gächter, 2002, pp. 980-995). Igualmente, los hallazgos de Henrich demuestran que las diferencias de grupo son más importantes que las características personales en la explicación de los comportamientos individuales. Incluso los experimentos realizados partiendo de la teoría de juegos ponen de evidencia que los individuos pueden comportarse de modo altruista incluso en juegos simples o de una única tirada. En este sentido, Nowak (2000, pp. 1773-1775) llega a afirmar que las respuestas humanas emocionales han sido configuradas por miles de años de convivencia en sociedades y grupos de pequeña escala y que nuestras interacciones nunca constituyen interacciones anónimas, con lo que los supuestos relacionados con un mercado impersonal no expresan el modo en que el hombre adopta las elecciones sociales.

Y lo que es más: tal como pone de relieve la Behavioral Decision Theory, deberíamos hablar de dos modos de racionalidad. Por una parte está el modo experimental, intuitivo, automático y natural, centrado en imágenes a las que se asocian sentimientos positivos o negativos a través de procesos de aprendizaje o experiencia. Esta heurística afectiva, en la que los individuos de modo intuitivo realizan juicios y toman decisiones, parece vinculada a la mayoría de los entornos habituales de comportamiento y resulta especialmente ajustada para responder a situaciones en las que la experiencia nos capacita para

12 Los trabajos de Kahneman y Tversky son paradigmáticos en esta línea de trabajo al poner en cuestión los fundamentos de la teoría de la utilidad (Kahneman y Tversky, 1979, pp. 263-291). 
anticipar los resultados agradables o desagradables de una determinada decisión. No obstante, este modo de razonamiento instrumental falla cuando los resultados no pueden ser anticipados en razón de experiencias previas. Esta última situación nos lleva a un segundo modo de racionalidad, la analítica, que ha sido tradicionalmente considerada por la Teoría Económica y que supone un proceso lógico de decisiones y valoraciones orientado a una acción más retardada, pero que puede resultar excepcional, ya que la mayor parte de las decisiones se asocian al cumplimiento de normas o a la ejecución de rutinas ${ }^{13}$.

Concretando estas divergencias en cinco rasgos:

1. En primer lugar, el presupuesto de egoísmo (no tuismo) carece de fundamento. Si bien es cierto que los seres humanos persiguen su propio interés, la pregunta realmente importante es en qué medida el interés particular constituye su principal preocupación. Diversas teorías en las ciencias conductuales tan reconocidas como el ciclo vital de Ken Wilber, los siete estadios de desarrollo de la moral de Piaget y Kohlberg, o la más conocida de la jerarquía de necesidades de Maslow, vienen a poner de manifiesto la existencia de una evolución temporal en los modelos de racionalidad práctica del hombre y sus prioridades ${ }^{14}$.

2. De igual manera, la pretensión de separación del mundo físico y humano es deficiente. Los seres humanos no pueden

13 Sobre el análisis de los modos divergentes de racionalidad resulta especialmente interesante el artículo de P. Slovic et al., 2002, pp. 329-342.

14 Estas tres teorías, las predominantes en los estudios sobre la evolución de prioridades valorativas, vienen a reconocer un proceso de desarrollo humano desde estadios prepersonales (autocentrados) en terminología de Wilber, en los que la preocupación prioritaria es la satisfacción de las necesidades básicas elementales (fisiológicas, de seguridad...) hacia estadios transpersonales, en los que la prioridad pasa a reconocimiento e incluso a las dimensiones trascendentes. En la misma línea, el trabajo de Kohlberg pone de manifiesto una evolución paralela en la moral desde los criterios de interés particular hasta los niveles calificables de proexistencia. Véase Wilber, 1996, y Maslow, 1971. 
ser comprendidos al margen de su medio humano y físico, es decir, se encuentran al menos parcialmente incrustados en la sociedad y la naturaleza.

3. La racionalidad humana, dista mucho del supuesto carácter mecanicista que se le supone y presenta respuestas más holísticas. De igual manera es preciso considerar la existencia de costes y límites en la habilidad de calcular, patologías psicológicas y emocionales generalizadas y la ineludible necesidad de aceptación social.

4. El carácter supuestamente irreflexivo del homo oeconomicus resulta fácilmente falseable. Toda forma de socialización conlleva un proceso de interiorización de valores morales, religiosos, sociales, que se traduce en una capacidad de interpretación de la bondad o maldad de las elecciones, esto es, la aparición de una racionalidad evaluativa.

5. Finalmente, el supuesto de inmutabilidad del homo oeconomicus es deficiente. El ser humano experimenta un proceso de cambios que afectan tanto a su visión de la realidad como a sus motivaciones.

En conclusión y recogiendo las propias palabras de Maslow, la Economía como ciencia "plantea una aplicación hábil, exacta y tecnológica de una teoría totalmente falsa de las necesidades y valores humanos, una teoría que sólo reconoce la existencia de necesidades materiales y de orden inferior» (Maslow, 1971, 310).

\subsection{Algunas propuestas divergentes}

Cada una de las distintas escuelas heterodoxas intenta dar una respuesta a las deficiencias observadas en la antropología del homo oeconomicus, proponiendo una visión del hombre más holística. Vamos a analizar muy brevemente cada una de estas orientaciones (Tomer, 2001, pp. 281-293) (Esquema I.1). 
Esquema I.1. Paradigmas antropológicos Alternativos

Homo oeconomicus-socialis
El hombre como ser social $\left\{\begin{array}{l}\text { Homo oeconomicus } \\ \text { Institucional } \\ \text { Influencia de las instituciones y normas sociales } \\ \text { Homo socioeconomicus } \\ \text { Influencia de la dimensión moral y emocional } \\ \text { La conciencia del nosotros } \\ \text { Economía humanista } \\ \text { Introducción de la dimensión evaluativa } \\ \text { Carácter eudemonista de la Economía } \\ \text { Escuela Austriaca } \\ \text { El ser humano como creador con capacidad } \\ \text { informativa limitada } \\ \text { Carácter cataláctico de los procesos sociales } \\ \text { La ética como un componente de la racionalidad } \\ \text { económica }\end{array}\right.$

Fuente: Elaboración propia.

\subsubsection{El «homo oeconomicus-socialis»}

Bajo este epígrafe pueden aglutinarse un importante conjunto de escuelas de pensamiento económico que van desde el materialismo marxista y los diversos historicismos a la Economía Humanista, pasando por la Economía Feminista, e incluso alguna que otra vertiente postkeynesiana. La característica común que podemos encontrar en todos ellos es el socio-idealismo.

En todas estas orientaciones aparece una doble dimensión: social e individual. Todo individuo busca su interés particular, pero simultáneamente es un ser social, dada su pertenencia a organizaciones e instituciones. Así, en determinadas circunstancias, los individuos parecen comportarse conforme a la racionalidad individualista del homo oeconomicus; sin embargo, los 
comportamientos más frecuentes resultan sub-racionales o reflejan los sesgos de alguna pertenencia social. Esta dimensión social es heterocentrada, comunal, dependiente y asociada a la cultura. Por tanto, el hombre combina un sentido cooperativo, comunal, con una tendencia a la competitividad. De igual manera, presenta una dimensión idealista, ya que tiene consciencia y una preocupación cuasi-natural por la justicia, la dignidad, el deber, la lealtad, etc. (O'Boyle, 1994, pp. 286-313). Dentro de este núcleo general hemos querido destacar al menos tres orientaciones.

\subsubsection{El «homo oeconomicus» institucional}

En esta versión, al contrario que la convencional, el comportamiento humano no tiene carácter inmutable, sino que sigue hábitos y reglas; por tanto, se encuentra fuertemente influido por instituciones, así como aprende de su experiencia social y personal. Consecuentemente, el comportamiento de este modelo se asienta sobre refuerzos institucionales, con lo que el homo oeconomicus institucional no constituye una realidad escindida del grupo de pertenencia y evoluciona al mismo tiempo que éste. Por otra parte, no puede calificarse como un maximizador de utilidad porque sus objetivos son determinados socialmente ${ }^{15}$, aunque sí resulta no-tuista, o guiado por el interés particular. Las teorías veblenianas son un claro ejemplo en este sentido (Veblen, 1974).

15 Puede encontrarse una excelente aproximación a esta orientación en Hodgson, 1998, pp. 166-192. Igualmente, en Whalen, 1996. 


\subsubsection{El homo «socio-oeconomicus»}

La visión de la socio-economía es totalmente heredera de la propuesta genérica anterior, pero su desarrollo epistémico ha llegado a constituir toda una escuela de pensamiento ya que la antropología subyacente a esta orientación deja en un segundo plano al hombre como decisor racional. Tal y como señala Etzioni: «El ser humano toma decisiones sobre la base de emociones y juicios de valor y sólo secundariamente sobre consideraciones lógicas y empíricas. Incluso, aunque pretenda tomar decisiones en el estrecho margen de la lógica, sus decisiones son todavía sub-racionales, producto de su capacidad limitada» (Etzioni, 1988, p. xi).

Esta escuela opta también por una división del hombre en dos dimensiones: por un lado el yo y por otro el nosotros. La dimensión del yo funciona de modo muy semejante a la propuesta convencional del homo oeconomicus; sin embargo, la dimensión del nosotros se encuentra orientada hacia los otros (la familia, la comunidad, la sociedad...) y en ella predominan tanto las obligaciones como los compromisos. Fruto de esta división, el comportamiento humano está determinado por ambos principios, frecuentemente en conflicto, aspecto que no puede ser representado por una única función de utilidad (Etzioni, 1998, pp. 539-549). Es destacable de igual manera que el modelo antropológico de esta escuela es reflexivo en el sentido de que es capaz de reflexionar sobre su propio comportamiento a la luz de sus valores morales.

De entre las propuestas alternativas de carácter holístico a la mainstream hemos querido destacar dos paradigmas alternativos. La propuesta humanista, aunque propiamente no constituye un paradigma alternativo, sino una corrección de la perspectiva materialista neoclásica en un sentido aristotélico; y el que subyace a la tradición austriaca, que introduce la ética como un componente esencial en el proceso de cooperación 
social que caracteriza la economía de mercado aportando una nueva perspectiva de análisis de los procesos de mercado partiendo de una visión del hombre no reduccionista.

\subsubsection{La Economía Humanista}

Entre las propuestas que reaccionan al paradigma antropológico tradicional está la denominada «Escuela Humanista». Esta orientación va a hacer también hincapié en una concepción creativa del ser humano y en su carácter finalista, recuperando una visión típicamente aristotélica para las ciencias sociales, pero sin rechazar las aportaciones de la Economía Neoclásica.

En primer lugar, el hombre es reconocido en su capacidad para reconstruir la sociedad y el mundo natural en conformidad con sus deseos; junto a ello, su finalidad última no puede traducirse en una función de la utilidad, sino que es el logro de un fin eudemonista, una "vida buena», a través de la persecución de unos objetivos intermedios. Esto es, se propone una recuperación del finalismo eudemonista. Tal y como señala J. Segal, la acción del hombre, tanto en la esfera privada como en la esfera pública, no puede ser explicada a menos que se plantee la existencia de un principio de sentido o comprensión de la naturaleza humana. Esto incluye una visión de la naturaleza humana, una definición de lo que constituye una vida buena y la delimitación de un proyecto humano (Segal, 1991, pp. 287-309).

Contraponiendo ambos paradigmas, la Economía Neoclásica presenta un único objetivo, el crecimiento, adoptando como uno de los supuestos básicos la insaciabilidad. Por el contrario, la Economía Humanista se alinea con propuestas filosófico-teológicas para adoptar como punto de partida la limitación de los propios deseos y del peso de los bienes y servicios convencionales con relación a la calidad de vida. Así, el homo sapiens de la Economía Humanista es, antes que utilitarista y un calculador «cazador de gangas», capaz de reflexionar sobre 
su propia condición de hombre y sobre aquello que desarrolla la naturaleza humana, y capaz, por tanto, de realizar elecciones responsables ${ }^{16}$.

Esta oposición de paradigmas también se ha expresado a través del conflicto entre reduccionismo y holismo, o, en expresión de Goeller, entre empty-world economics y full-world economics (Goeller y Weinberg, 1978, pp. 1-11; Daly y Cobb, 1989), aludiendo al hecho de que esta orientación abarca aspectos que la economía convencional situaría fuera de la ciencia, o, más propiamente, filosóficos o ecológicos. Frente a éste, la mainstream, bajo la pretensión de objetividad, habría limitado el estudio a aspectos puramente técnicos, abandonando objetivos más ambiciosos referidos a la vida en un sentido amplio. Tal y como señala D. Eberle, el siglo XX ha transformado el hombre moral en el hombre económico y psicológico; si queremos volver a construir algún modo de sociedad, tendremos que recuperar una visión del hombre como ser moral y con un poder de agenda ético. De este modo, aquellos aspectos definidores del ser humano son ante todo la capacidad de reflexión, simpatía, compromiso con un ideal y libertad, aspectos no considerados en la Economía convencional, para la que toda acción tiene como única motivación el interés particular.

Sintetizando, estas divergencias pueden reducirse a los siguientes aspectos. En primer lugar, frente a una definición de Economía como una ciencia de elección racional entre recursos escasos ante deseos ilimitados, la vertiente humanista toma como punto de partida la discusión sobre los bienes y servicios necesarios para el hombre. Aunque esta pregunta no pueda ser respondida, su planteamiento es imprescindible con el fin de definir lo que constituye la «vida buena». La ciencia plantearía entonces una toma de decisiones eficientes usando recur-

16 Sobre la contraposición de ambos paradigmas puede verse un resumen en Foldvary, 1996, 120-128. 
sos escasos, pero de tal forma que el mayor número de personas posible, ahora y en el futuro, pueda alcanzar la excelencia. Consecuentemente, la Economía tiene como fin último una doble tarea: lograr que cada hombre disponga de una suficiente cantidad de bienes y servicios y, junto a ello, contribuir al logro de una vida humana en plenitud.

En tercer lugar está el problema del mal, ignorado en la Economía; es uno de los objetos de estudio, en particular, el papel de las instituciones económicas, sociales y políticas en su eliminación ${ }^{17}$.

En cuarto lugar, dado que los hombres disponen de capacidad para distinguir entre lo que constituye un auténtico cumplimiento de la naturaleza humana y lo que no, así como de libertad para seguir o no sus deseos auténticos, lo que precisan los sujetos no es la satisfacción de sus deseos, sino disponer de un conjunto de objetivos acorde con el ser del hombre. Como resultado, dentro de la Economía Humanista los capítulos dedicados al comportamiento del consumidor revisten una importancia especial y se centran en el análisis del origen de los deseos, completándolo con extensas discusiones sobre la finalidad del consumo como mera búsqueda hedonista o la consecución de una completud vital o la excelencia del ser.

Finalmente, esta escuela pone en cuestión la supuesta objetividad científica de la teoría económica convencional, otorgando mayor importancia al papel de la experiencia y la intuición en la construcción de la ciencia. Desde su punto de vista, toda teoría debería ser cuestionada sobre su significado para el ser humano y no meramente validada por sus resultados empíricos. Como consecuencia, el ser humano y la experiencia humana, tanto en el análisis de la antropología como de la sociología o de la psicología, son incorporados como fuente de información.

17 En palabras de Daly, la economía neoclásica ha evadido constantemente el problema del mal recurriendo a la metáfora progresista del salto adelante. Por tanto, los problemas morales serían eliminados por el crecimiento (Daly, 1979, 37-59). 
Asimismo, los economistas humanistas sienten una preocupación especial por la capacidad de la economía para promocionar un fuerte sentido de comunidad, dado que es a través de la pertenencia a estas instituciones como adquirimos el conocimiento de lo que significa ser hombre.

Pese al interés indudable de esta orientación, la Economía Humanista peca de un notable voluntarismo ético, para caer en los mismos errores en los que cae la ética en su relación con la Economía; es decir, se limita a aplicar una corrección moral al funcionamiento de la economía, pero desde una perspectiva ajena al propio hecho económico.

\subsubsection{La Escuela Austriaca}

Tal y como vimos, la teoría económica convencional se basa en la aplicación de un concepto estrecho de racionalidad, para el que cualquier proceso de toma de decisiones se limita a la aplicación de un criterio de maximización de la utilidad teniendo en cuenta los costes y beneficios derivados de cada alternativa. Esta perspectiva supone una pérdida de relevancia de la dimensión ética del actuar, ya que la simple búsqueda de las consecuencias más beneficiosas podría parecer un criterio suficiente. Consecuentemente, en la tradición neoclásica las consideraciones relativas a los principios éticos como guía del comportamiento humano perdían relevancia bajo la pretensión de lograr una ciencia universal del comportamiento asentada sobre un único principio, el criterio maximizador de la utilidad esperada.

Sin embargo, el hombre no puede disponer de toda la información, ni de la capacidad necesaria para ese proceso de optimización (Huerta de Soto, 1999, 150 ss.). Ante este problema, la Escuela Austriaca propone un paradigma alternativo al neoclásico, asentado en tres pilares. En primer lugar, el estudio del ser humano como creador y del mercado como institución que coordina la información dispersa. En segundo lugar, el 
carácter evolutivo y, por tanto, dinámico de las creaciones sociales, desde el lenguaje a la economía, incluyendo, lógicamente, la moral. Éstos no son un producto de construcciones racionales individuales, sino la consecuencia natural de la participación de infinidad de actores, aglutinando ingentes cantidades de información. Finalmente, la ética vuelve a ocupar un lugar esencial como guía del actuar individual.

Partiendo de que el proceso social es una realidad constituida a través de la interacción de multitud de individuos, cada uno de los cuales dispone de una capacidad creativa innata, resulta del todo imposible calcular los costes y beneficios que se derivan de una determinada decisión. Esto implica que la coordinación, lejos de fundarse en el cálculo, lo hace sobre los principios morales, con lo que reviste rasgos dinámicos y no sería fruto de un racionalismo constructivista. Consiguientemente, la eficiencia, comprendida como coordinación, surge del comportamiento de seres humanos guiados por directrices morales (Huerta de Soto, 1995, 25-32), y, por tanto, no cabe la existencia de relaciones de intercambio (trade off) entre Ética y Economía, típicas de la Economía Neoclásica, sino, por el contrario, de complementariedad. En esta aparente relación de conflicto, para la Ética, la Economía y en particular el mercado, es objeto de corrección, en tanto que para la Economía, la Ética es una institución normativa ajena al mercado. Así, por ejemplo, construcciones características como la denominada «Economía del Bienestar», habrían dado lugar a nociones vacías como la de «justicia social», bajo la cual son juzgados los procesos sociales al margen de los comportamientos individuales de los que participan en ellos ${ }^{18}$.

De este modo, el consecuencialismo tradicional en el utilitarismo no es capaz de afrontar el problema de la acción huma-

18 La crítica al concepto de «justicia social» la podemos encontrar en Hayek, 1988. Igualmente, en I. Kirzner, 1989. 
na, con lo que las normas de comportamiento apriorísticas asentadas en principios éticos constituyen pilotos automáticos necesarios o formas de precoordinación de las actuaciones individuales, esto es, facilitan la propia coordinación e intercambio de información que genera el mercado. Con lo que existe una clara imbricación entre la teoría económica y la ética social, lo que no implica que la teoría económica pueda llegar a formular por sí sola los planteamientos morales, sino que permite facilitar el razonamiento lógico-deductivo de la ética, evitando los errores ocasionados por los supuestos irreales de plena información y las insuficiencias del concepto de racionalidad. (Huerta de Soto, 1996, pp. 101-132).

En definitiva, el desarrollo teórico de la mainstream en Economía, centrado en la noción de eficiencia estática, tiene como punto de apoyo una concepción antropológica y sociológica errónea y heredera del psicologismo y cientifismo decimonónico ${ }^{19}$, donde uno de los principales problemas es la supuesta escisión entre comportamiento económico y ético. Para la tradición austriaca, la Economía como sistema se asienta sobre una cadena lógica de principios elementales: 1) La propiedad privada, como principal incentivo al desarrollo de la creatividad; 2) la libertad de disposición de esa propiedad; 3) la competencia en el mercado como sistema de intercambio dinámico de información, y 4) división del trabajo y cooperación social, puesto que la sociedad es sólo la combinación de individuos para el desarrollo de una tarea común (Von Mises, 1995, 173203). Dentro de este último componente, la ética es el fundamento de la cooperación.

Para esta escuela, por tanto, la teoría económica es muy próxima a la ética social y existe una auténtica interrelación entre

19 Concretamente, los principales autores de esta escuela rechazan la construcción de una ciencia económica que se limite a imitar el paradigma matemático típico de la Física. 
ambas (Kirzner, 1994). Esta última propuesta, donde Economía y Ética aparecen claramente integradas, nos puede servir como punto de partida para una revisión del paradigma antropológico vigente.

\section{CONCLUSIONES: EL HOMBRE ÉTICO}

«El principio de la acción es la elección y el de la elección el deseo y la elección de un fin. Por eso, ni sin entendimiento y reflexión ni sin disposición moral hay elección. La elección es inteligencia deseosa o deseo inteligente, y esta clase de principio es el hombre.»

Aristóteles, Ética a Nicómaco

Conforme a lo analizado, los restrictivos supuestos antropológicos sobre los que se construye el homo oeconomicus dejan abierta la posibilidad de un amplio conjunto de alternativas... Sin embargo, es conveniente centrarse en un único planteamiento holístico que denominamos hombre ético. Este concepto articula una visión notablemente más amplia y potencialmente más realista de la naturaleza humana, y posee una larga tradición en la historia de la reflexión filosófica sobre la economía ${ }^{20}$, en particular dentro de la última escuela mencionada.

El concepto de hombre ético recoge el hecho de que los individuos no sólo difieren en preferencias respecto a bienes y servicios, sino también en objetivos con los que se sienten comprometidos, en normas personales, en su capacidad de afrontar decisiones complejas, en el conocimiento y en las orientaciones que han heredado de experiencias previas, en su pertenencia a una comunidad, etc.

20 Podemos encontrar un sin fin de antecedentes, entre los que cabe destacar como cita habitual las obras de Knight, 1933, y Macfie, 1936, 1946. 
Esta perspectiva holística proporciona un marco analítico mucho más rico que el explicitado por la teoría económica convencional para investigar la actuación de grupos, tanto a nivel micro como macro y desde el mercado hasta el funcionamiento del sistema político o del Estado, y permitirá generar una vía de enlace entre la visión generalizada dentro de la Economía, las propuestas alternativas y otras ciencias sociales.

Veamos ahora los aspectos fundamentales de esta definición.

El hombre ético, al igual que en alguna medida el hombre económico, es finalista y racional, si bien más semejante en este aspecto al hombre de la escuela austriaca ${ }^{21}$. No obstante, sus fines están fijados de un modo muy general y sólo en situaciones concretas se traducen en objetivos más próximos a los económicos. Además, estos fines generales no tienen naturaleza autónoma sino que pueden estar influidos por los grupos sociales a los que pertenecen los individuos y, en último término, están sujetos a una racionalidad evaluativa.

En cuanto a la racionalidad, el hombre ético no es identificable con el modelo que supone la Economía Neoclásica, dado que no se enfrenta a problemas hipotéticos perfectamente especificados, sino con problemas complejos en los que el análisis heurístico intuitivo es un complemento esencial del análisis formal. Esto le conduce a descansar fuertemente en el control de rutinas ante el problema de la toma recurrente de decisiones, que sólo serán reconsideradas cuando emerge un problema concreto en el que falla el planteamiento de la norma. Igualmente el hombre ético se encuentra vinculado a un grupo de pertenencia $\mathrm{y}$, por tanto, se caracteriza por recibir la identidad personal y formar sus preferencias a través de su incorporación a un colectivo. En otros términos, su racionalidad tiene una dimensión comunitaria y cooperativa.

21 En este sentido, además de las citadas, puede resultar esclarecedora Kirzner, 1986. 
Por otro lado, al adoptar la perspectiva del «nosotros», el hombre ético presenta una visión más amplia de la naturaleza del problema, analizando aspectos del medio y consecuencias grupales que el homo oeconomicus ignora, ya que éste optimiza su comportamiento sobre modelos que fallan en endogeneizar la respuesta de los restantes miembros del grupo. Así, cuando el hombre ético «juega» con un oponente igualmente racional, desarrolla estrategias cooperativas que conllevan formas recíprocas de deber.

En definitiva, la Ética es indudablemente relevante para la Economía, no sólo en su dimensión normativa, donde es particularmente evidente por su carácter evaluativo, sino también en su dimensión positiva porque está incidiendo constantemente en las actuaciones económicas de los individuos. No obstante, esta búsqueda no debe remontarse hasta las raíces, lo que sería en el sentido aristotélico definir lo que constituye una «vida buena», sino analizar cómo la adhesión a ciertas normas está incidiendo en los comportamientos individuales $\mathrm{y}$, consecuentemente en el logro de objetivos económicos. Los éxitos del capitalismo, incluido la posibilidad de alcanzar mayores niveles de vida, no podrían explicarse de no tener en cuenta el trabajo en equipo, la coordinación, la confianza que vienen a mostrar el papel clave de los códigos de comportamiento. Por tanto, aunque no se trata de una nueva discusión de fines, sí exige una discusión sobre el realismo de los constructos y simplificaciones a los que nos tiene acostumbrada nuestra ciencia, en particular el que conocemos como homo oeconomicus. Así, la reflexión sobre la interrelación entre Ética y Economía es el modo de introducir realismo o acercar la economía al proceso real de toma de decisiones en el mercado (Koslowski, 1991, 9-14). 
Esquema I.2. CONTRASTACión DEL HOMO OECONOMICUS FRENTE AL ETHICUS

\begin{tabular}{|c|c|}
\hline Homo oеconomicus & Homo ethicus \\
\hline $\begin{array}{l}\text { Racional en un sentido instrumental } \\
\text { - Comportamientos sistemáticos } \\
\text { - Adaptativos } \\
\text { - Discernibles }\end{array}$ & $\begin{array}{l}\text { Racionalidad finalista } \\
\text { — Racionalidad heurística compleja } \\
\text { Heurística efectiva } \\
\text { - Comportamientos inerciales }\end{array}$ \\
\hline $\begin{array}{l}\text { Conjunto de preferencias } \\
\text { completo y totalmente ordenado } \\
\text { - Deus ex machina- }\end{array}$ & $\begin{array}{l}\text { Sistema complejo de preferencias } \\
\text { - Metapreferencias (Sen) } \\
\text { - Simpatía y compromiso } \\
\text { - Múltiples funciones de utilidad } \\
\quad \text { (Etzioni) }\end{array}$ \\
\hline Información perfecta & $\begin{array}{l}\text { Información imperfecta } \\
\text { - Procesos de aprendizaje e imitación }\end{array}$ \\
\hline Perspectiva estática de equilibrio & Perspectiva dinámica \\
\hline $\begin{array}{l}\text { Carácter individualista } \\
\quad \text { (Leader) }\end{array}$ & $\begin{array}{l}\text { Racionalidad grupal y cooperativa } \\
\text { Comportamientos: } \\
\text { - Reputacionales } \\
\text { - Simpatías }\end{array}$ \\
\hline
\end{tabular}

Fuente: Elaboración propia.

Lo planteado en el presente artículo nos permitiría afirmar la necesidad de una revisión del modelo antropológico vigente en la Teoría Económica teniendo en cuenta los resultados de las ciencias conductuales. Dicha revisión nos conducirá a un replanteamiento tanto de la teoría como la práctica de la ciencia económica y, en particular, de la Política Económica.

En primer lugar, la revisión del paradigma del homo oeconomicus debería incorporar aspectos tales como la racionalidad limitada y sus derivaciones (capacidad de aprendizaje, imitación, inercias...). En este sentido, la línea más interesante de la agenda de investigación es el estudio de la caracterización de los agentes a través de la comprensión del conocimiento humano, atendiendo a aspectos tales como la heterogeneidad cultural y valorativa de los individuos e incluso la dimensión ética 
y emocional. Indudablemente, estos modelos conductuales son más complejos que los actuales pero potencialmente más realistas, al incorporar conclusiones de otras ciencias sociales como la psicología (Thaler, 2000, pp. 133-141).

Aquellos modelos que sean capaces de incorporar el altruismo y otros aspectos del proceso de toma de decisiones conducirán necesariamente a mejores predicciones; esto es, la teoría económica debe partir del comportamiento, tal y como se expresa, no de supuestos que hagan el comportamiento irreal pero matemáticamente tratable.

\section{BIBLIOGRAFÍA}

Akerlof, G.A. (1983), «Loyalty Filters», American Economic Review, 73, 54-63.

Arrow, K. (1985), «The Economics of Agency», en J.W. Pratt y J. Zeckhauser, Principals and Agents, Boston, Havard Business School, 37-55.

Arrow, K. y Debreu, G. (1954), «Existence of Equilibrium for a Competitive Economy», Econometrica 22, 265-290.

Becker, G. (1976), The Economic Approach to Human Behaviour, Chicago, University of Chicago Press.

BeCKer, G. y Stigler, J. (1977), «De gustibus non est disputandum», American Economic Review, 67, 76-90.

Blaug, M. (1980) La metodología de la economía, Madrid, Alianza.

Boland, L.A. (1982), The Foundations of Economic Method, London, Allen and Unwin.

CAmerer, C.F. (1997), «Progress in Behavioural Game Theory», The Journal of Economic Perspectives 11, 4, 167-188.

CAson, M.C. (1990), «Economic Man», en J. Creedy (ed.), Foundations of Economic Thought, Oxford, Blackwell.

DALY, H. (1979), «Ethical Implications of Limits to Global Development», en W. Finnin, The Morality of Scarcity: Limited 
Resources and Social Policy, Batton-Rouge, Lousiana State University Press, 37-59.

Daly, H. y Совв, J. (1989), For the Common Good: Redirecting the Economy Towards Community; The Environment and a Sustainable Future, Boston, Beacon Press.

Davidson, D. (1980), "Actions, Reasons and Causes», en D. Davidson, Essays on Actions and Events, New York, Oxford University Press, pp. 3-20.

Dimand, M.A. y Dimand, R.W. (1991), «Moral Sentiments and the Marketplace», en W. Barber, Preclassical, Classical and Marxian Economics, Chelteham, E. Elgar, pp. 61-75.

Etzioni, A. (1998), «The Methodology of Socio-Economics», Journal of Socio-Economics, 27, 5, 539-549.

- (1988), The Moral Dimension, Nueva York, Free Press.

Fehr, E. y GÄChter (2002), "Cooperation and Punishment in Public Goods Experiments», American Economic Review, 90, 980-995.

Foldary, F.E. (1996), Beyond Neoclasical Economics, Cheltenham, Edgar Elgar.

Friedman, M. (1952), Essays in Positive Economics, Chicago, University of Chicago Press.

Goeller, H. y WeinBerg, A. (1978), «The Age of Substitutability», American Economic Review, 68, 1-11.

Goldsmith, M. (1985). Private Vices, Public Benefits: Bernard Mandeville's Social and Political Thought, Cambridge, Cambridge University Press.

GonZÁLEZ, W.J. (2002). «Análisis de la racionalidad y planteamiento de la predicción en economía experimental», en W.J. González, Enfoques filosófico-metodológicos en economía, Madrid, Fondo de Cultura Económica.

- «Racionalidad y economía: de la racionalidad de la economía a la racionalidad de los agentes económicos», en W.J. González (ed.), op. cit., p. 75.

- (2004), Racionalidad, historicidad y predicción en H. Simon, A Coruña, Netbiblo. 
GuI, B. (2000), «Beyond Transactions: On the Interpersonal Dimension of Economic Reality», Annals of Public and Cooperative Economics, 71, 2, 139-169.

Hausman y McPherson (1993), «Taking Ethics Seriously», Journal of Economics Literature, 31, 2, 671-731.

HayeK, F.A. (1988), El espejismo de la justicia social, vol. II: Derecho, legislación y libertad, Madrid, Unión editorial, 2. a edición.

Hirschman, A. (1986), Interés privado y acción pública, México D.F., FCE.

Hodgson, G.M. (1988), Economics and Institutions, Oxford, Blackwell.

- (1998), «The Approach of Institutional Economics», Journal of Economic Literature 36, 1, 166-192.

Hollis, M. y Nell, E. (1975), Rational Economic Man: A Philosophical Critique of Neoclassical Economics, Cambridge, Cambridge University Press.

Huerta de Soto, J. (1996), «Socialismo, corrupción ética y Economía de Mercado», en L. Núñez Ladeveze (ed.), Madrid, Noesis, pp. 101-132.

- (1995), «Estudio preliminar» a I. KIRZNER, Creatividad, capitalismo y justicia distributiva, Madrid, U. Editorial, pp. 25-32.

- (1999), «The Ethics of Capitalism», Journal of Markets and Morality, II, 2, 150-151.

- (2004), «La teoría de la eficiencia dinámica», Procesos de Mercado: Revista Europea de Economía Política, vol. I, n. ${ }^{\circ}$ 1, pp. 11-71.

HeNRICH, J. et al. (2001), «In Search of homo oeconomicus: Behavioral Experiments in 15 Small-Scale Societies», American Economic Review, 91, 73-79.

Kahneman, D. y TVersky, A. (1979), «Prospect Theory: An Analysis of Decision under Risk», Econometrica, 47, 2, pp. 263291.

KIRZNER, I.M. (ed.) (1986), Subjectivism, Intelligibility and Economic Understanding, London, Macmillan. 
- (1989), «Some Ethical Implications for Capitalism of the Socialist Calculation Debate», en E. Frankel et al., Capitalisms, Oxford, Blackwell.

- (1994), Hayek Social and Political Thought, Oxford, Clarendon Press.

Knetch, J. (1992), «Preferences and Nonreversibility of Indiference Curves», Journal of Economic Behavior and Organization, 17, 129-139.

KNIGHT, F.H. (1993), The Ethics of Competition, London, Allen and Unwin.

KosLowski, P (ed.) (1991), Ethics in Economics, Business and Economic Policy, Berlin, Springer-Verlag.

Loomies, G. y TAYLORD, C. (1992), «Non Transitive Preferences over Gains and Losses», Economic Journal, 102, 357-365.

Macfie, A.L., An Essay on Economy and Value: Being an Inquiry into the Real Nature of Economy, London, Macmillan, 1936, y Economic Efficiency and Social Welfare, London, Oxford University Press, 1946.

MACNAIL, I. (1985), «Reflections on Relational Contracts», Journal of Institutional and Theoretical Economics, 141, 541-546.

Martínez Velasco, L. y Martínez Hernández, J. (1994), La casa de cristal, hacia una subversión normativa de la economía, Madrid, Fundamentos.

Maslow, A. (1971), The Farther Reaches of Human Nature, Nueva York, Penguin.

Mises, L. Von (1995), La Acción Humana, Madrid, Unión Editorial.

NoWAK, M. et al. (2000), "Fairness versus Reason in the Ultimatum Game», Science 289, 1773-1775.

O’Boyle, E. (1994), «Homo Socio-Economicus: Foundational to Social Economics and the Social Economy», Review of Social Economy, 52, 3, 286-313.

Oкun, А. (1981), Prices and Quantities, Oxford, Blackwell.

RESCHER, N. (1988), Rationality, a Philosophical Inquire into the Nature and the Rationale of the Reason, Oxford, Clarendon Press. 
Segal, J. (1991), "Alternative Conceptions of the Economic Realms», en R.M. Coughlin (ed.), Morality, Rationality and Efficiency: New Perspectives in Socio-Economics Nueva York, Sharpe, pp. 287-309.

SElten, R. (1994), «In Search of a Better Understanding of Economic Behaviour», en A. Heertje, The Makers of Modern Economics, Londres, Harvester.

Selten, R. y Ockenfels, A. (1998), «An Experimental Solidarity Game», Journal of Economic Behavior and Organization, 34, 4, 517-539.

SEN, A. (1977), «Rational Fools: A Critique of the Behavioural Foundations of Economic Theory», Philosophy and Public Affairs, 6, 324-350.

- (1985), «The Moral Standing of the Market», en E.F. Paul et al. (eds.), Ethics and Economics, Oxford, Blackwell, 1-19.

SHelling, G. (1960), The Strategy of Conflict, Cambridge, Harvard University Press.

Simon, H. (1983), Reason in Human Affairs, Stanford University Press.

- «From substantive to procedural rationality», 130-131.

- (1993), «Altruism and Economics», American Economic Review, 83, 156-161.

- (2000), «Bounded Rationality in Social Science», Mind and Society, 1, 25-39.

Slovic, P. et al. (2002), «Rational Actors or Rational Fools: Implications of the Affect Heuristic for Behavioral Economics», The Journal of Socioeconomics, 31, 329-342.

Sмiтн, V. (1998), «Theory, Experiment and Economics», Journal of Economic Perspectives, I, 3, 151-168.

THALER, R.H. (2000), «From homo oeconomicus to homo sapiens», Journal of Economic Perspectives, 14, 1, pp. 133-141.

ToMER, J.F. (2001), «Economic Man vs. Heterodox Men», Journal of Socioeconomics, 30, 281-293.

Veblen, T. (1974), Teoría de la clase ociosa, Fondo de Cultura Económica, México. 
Whalen, Ch. (1996), The Institutional Approach to Political Economy, en F. Foldvary (ed.), Beyond the Neoclasical Economics, Brookfield, Elgar.

Wilber, K. (1996), The Atman Project, a Transpersonal View of Human Development, Wheaton, Quest Books.

Wiles, P. y Routh, G. (eds.) (1984), Economics in Disarray, Oxford, Blackwell.

ZAMAGNI, S. (1989), «Sulla cooperazione tra scienza economica e etica: ragioni, problemi, prospettive», Orientamenti Sociali, I, 65-77.

- (1994), «Sulla relazione tra economia e filosofia: argomenti per un ampliamento del discorso economico", Economia Politica, XI, 281-296. 